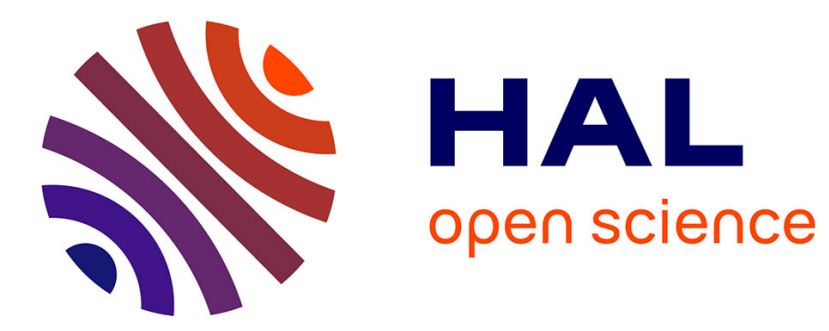

\title{
Solid-state synthesis of cyclo LD-diphenylalanine: A chiral phase built from achiral subunits
}

\author{
Ariel Pérez-mellor, Katia Le Barbu-Debus, Anne Zehnacker
}

\section{To cite this version:}

Ariel Pérez-mellor, Katia Le Barbu-Debus, Anne Zehnacker. Solid-state synthesis of cyclo LDdiphenylalanine: A chiral phase built from achiral subunits. Chirality, 2020, 32 (5), pp.693-703. 10.1002/chir.23195 . hal-02571724

\section{HAL Id: hal-02571724 \\ https://hal.science/hal-02571724}

Submitted on 26 May 2020

HAL is a multi-disciplinary open access archive for the deposit and dissemination of scientific research documents, whether they are published or not. The documents may come from teaching and research institutions in France or abroad, or from public or private research centers.
L'archive ouverte pluridisciplinaire HAL, est destinée au dépôt et à la diffusion de documents scientifiques de niveau recherche, publiés ou non, émanant des établissements d'enseignement et de recherche français ou étrangers, des laboratoires publics ou privés. 


\section{Solid-State Synthesis of cyclo LD-Diphenylalanine: a Chiral Phase Built from Achiral Subunits}

\begin{abstract}
The solid-state structure of LL/DD or LD/DL diphenylalanine diluted in $\mathrm{KBr}$ pellets is studied by infrared absorption (IR) and vibrational circular dichroism (VCD) spectroscopy. The structure depends on the absolute configuration of the residues. The natural LL diphenylalanine exists as a mixture of neutral and zwitterionic structures, depending on the humidity of the sample, while mostly the zwitterion is observed for LD diphenylalanine whatever the experimental conditions. The system undergoes spontaneous cyclisation upon heating at $125^{\circ} \mathrm{C}$, resulting to the formation of a diketopiperazine (DKP) dipeptide as the only product.
\end{abstract}

The reaction is faster for $L D$ than for $L L$ diphenylalanine. As expected, LL and DD diphenylalanine react to form the LL and DD enantiomers of cyclo diphenylalanine. Interestingly, the DKP dipeptides formed from the LD or DL diphenylalanine show unexpected optical activity, with opposite VCD spectra for the products formed from the LD and $\mathrm{DL}$ reagents. This is explained in terms of chirality synchronization between the monomers within the crystal, which retain the symmetry of the reagent, resulting to the formation of a new chiral phase made from transiently chiral molecules.

Ariel Pérez-Mellor, ${ }^{*[a]}$ Katia Le Barbu-Debus, ${ }^{[a]}$ and Anne Zehnacker ${ }^{*[a]}$

Keywords: chirality synchronization; Vibrational circular dichroism (VCD); diketopiperazine (DKP); peptides; vibrational spectroscopy

\section{Introduction}

Oligopeptides are important building blocks for the synthesis of supramolecular structures due to their self-assembly capabilities. ${ }^{1-8}$ The wealth of nanostructures formed opens a wide range of applications in the field of biosensors, organic semiconductors, and drug delivery. ${ }^{9-12}$ In particular, nanostructures formed from the Alzheimer's $\beta$-amyloid linear dipeptide diphenylalanine show a large degree of polymorphism. They have been extensively studied theoretically ${ }^{13-18}$ and experimentally. ${ }^{19} 20$ The balance between dispersive interactions among aromatic substituents and electrostatic interactions between the polar termini results in the formation of very stiff nanotubes consisting of a cyclic structure containing six molecules. The packing of these rings determines a supramolecular structure with chirality defined by that of the monomer, namely a left-handed helix for the natural LL diphenylalanine. The crystallographic study of these nanotubes suggests that their structure is the same as that of the single-crystal structure. Water has been suggested to play a role in the stabilization process. ${ }^{15}$ The molecules are indeed in their zwitterion form and are bound by strong interactions between the $\mathrm{COO}^{-}$and $\mathrm{NH}_{3}{ }^{+}$termini; the zwitterions being stabilized in aqueous environments. ${ }^{21}$ The benzene rings are in a T-shape configuration. Such a geometry is reminiscent of that observed in diphenylalanine or capped diphenylalanine isolated in the gas phase under supersonic jet conditions. ${ }^{22,23}$

Diphenylalanine, like other dipeptides built on aromatic residues, spontaneously undergo intramolecular peptide bond formation. ${ }^{5,24-27}$ The reaction product is a cyclic dipeptide called diketopiperazine (DKP). In particular, diphenylalanine nanotubes thermally cyclize to DKP structures. ${ }^{28}$ DKP peptides find applications as antivirals, antiparasitics, anticancer agents $^{29-31}$ or as catalysts. ${ }^{32,33}$

Unlike linear dipeptides, DKP containing dipeptides do not possess a terminal charged group and their crystal structure mainly involves $\mathrm{NH}$...OC hydrogen bonding interactions. The cyclo diphenylalanine crystal consists indeed of ladder-like structures bridged by a double $\mathrm{NH}$... $\mathrm{O}=\mathrm{C}$ hydrogen bond. ${ }^{34}$ The sub-unit of the crystal has a structure identical to that observed in the gas phase, with one aromatic ring folded over the DKP ring in a flagpole position and the other extended. ${ }^{22}$ Fourier Transform Infra-Red (FTIR) and Vibrational Circular Dichroism (VCD) spectroscopy of cyclo diphenylalanine powder in $\mathrm{KBr}$ pellets indicate that the system can be described as a dimer bridged by the same double hydrogen bond interaction as observed in the single crystal. ${ }^{35}$

Most studies conducted so far are devoted to dipeptides of natural absolute configuration $L$. The influence of the $L$ or $D$ absolute configuration of the residues on the self-assembly propensity of oligopeptides has been studied by electronic circular dichroism. ${ }^{36}$ Tripeptides based on $L$ or $D$ phenylalanine have been studied by circular vibrational dichroism and $\gamma$-turn nanostructures have been observed for L-Phe-L-Phe-D-Phe. ${ }^{37}$ We have recently compared the structure of jet-cooled diphenylalanine, denoted as LL hereafter, and cyclo diphenylalanine, denoted as $\mathrm{C}-\mathrm{LL}$, to that of their diastereomer LD diphenylalanine (LD) and cyclo LD diphenylalanine (c-LD). Conformer-selective vibrational spectroscopy compared to quantum chemical calculations indicates that the structural differences between the two diastereomers are small. ${ }^{22,38,39} \mathrm{C}$ $\mathrm{LL}$ and C-LD both show a folded-extended conformation, with one of the aromatic ring folded over the dipeptide frame and the other one extended outwards. C-LL and C-LD differ by the strength and the nature of $\mathrm{CH} \ldots \pi$ or $\mathrm{NH} \ldots \pi$ interactions. The most stable structure of linear LL and LD diphenylalanine both involve an extended structure of the peptide chain, with free $\mathrm{NH}_{2}$ and $\mathrm{COOH}$ groups at the termini. A bifurcated hydrogen bond takes place from the amide $\mathrm{NH}$ as a donor to both the acid $\mathrm{CO}$ and the $\mathrm{NH}_{2}$ group. $\mathrm{LL}$ and $\mathrm{LD}$ moistly differ by the position of the aromatic rings. Similar minor differences were 
observed for the protonated systems or the alkali-core complexes isolated in an ion trap..$^{40-42}$

In this work, we extend our FTIR and VCD studies on C-LL to its linear dipeptide precursor LL, as well as their diastereomers LD and C-LD. (Figure 1).

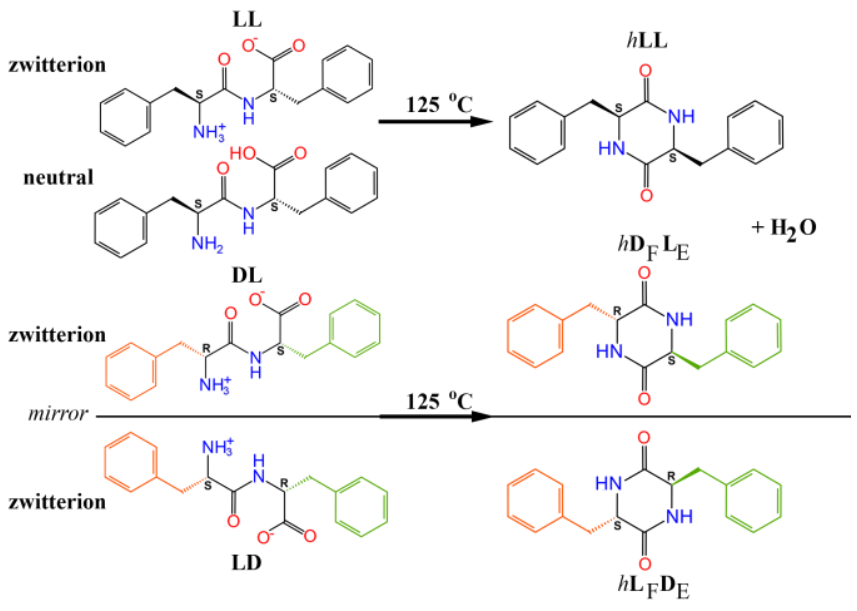

FIGURE 1 Systems under study and thermal reaction scheme. The colored residues simulate the asymmetric positions of the benzene rings (see text).

This work aims at comparing the solid-state structure of the powder of each diastereomer cyclo diphenylalanine and linear diphenylalanine. The presence of aromatic rings may influence the binding pattern by competing with the hydrogen-bond formation and we will compare the IR spectra of c-LD and c-LL at the light of this competition. While the stable form of most amino acids in the solid is a zwitterion, the oligopeptides can be either neutral or zwitterion, depending on the length of the peptide, the nature of the residue, and the environment, in particular presence of water. In what follows, we will also get information on the competition between neutral and zwitterion forms of LL and LD from their vibrational spectroscopy.

The second part of this work is devoted to the in situ synthesis of cyclo diphenylalanine by heating the $\mathrm{KBr}$ pellet and to the study of the cyclo diphenylalanine crystal obtained thereby. In solution, the reaction seems to proceed in the neutral dipeptide and not in the zwitterion. ${ }^{43}{ }^{44}$ The synthesis of DKP on a solid support has also been described. ${ }^{45}$ However, despite the existence of numerous studies on dipeptide cyclisation, the reaction has not been studied yet in $\mathrm{KBr}$ pellets.

\section{Materials and Methods}

\section{Sample Preparation}

The dipeptides (98\% purity) were purchased from GeneCustLuxembourg and Novopep Limited (Shanghai - China) and used without further purification. $\mathrm{KBr}$ pellets of the samples were prepared in the following way: first, $10 \mathrm{mg}$ of the studied molecules diluted with $3.5 \mathrm{~g}$ of $\mathrm{KBr}$ were carefully grinded in a mixer mill (MM 400 Retsch) at $20 \mathrm{~Hz}$ during $1 \mathrm{hr}$. This procedure avoids the formation of large crystals $(>5 \mu \mathrm{m}$ ) that could induce birefringence. The pellets were then made by pressing $150 \mathrm{mg}$ of this mixture up to 7 tons using a manual hydraulic press. Finally, the pellets were smoothly heated up to $80^{\circ} \mathrm{C}$ for 24 hours to eliminate most of the water.
A mass spectrum was recorded for each pellet after the thermal reaction, to check the nature of the products. The pellets were dissolved into a mixture of methanol and water (50:50) to form a $1 \mathrm{mM}$ stock solution. The $\mathrm{pH}$ of the solution was neutral. It was shown in our previous study that the LL or LD linear diphenyl alanine is neutral and not zwitterionic in the gas phase. ${ }^{40}$ The potassium core molecular complexes were generated by electrospraying a $100 \mu \mathrm{M}$ solution obtained by diluting the stock solution. The mass spectrum was recorded in a $7 \mathrm{~T}$ Fourier Transform Ion Cyclotron Resonance (FTICR) hybrid mass spectrometer (Bruker, Apex Qe) at the mass spectrometry platform SMAS of the Laboratoire de Chimie Physique (University Paris-Sud).

\section{Attenuated Total Reflection (ATR) - Fourier-Transform Infrared (FTIR) Spectroscopy}

The ATR-FTIR spectra were performed using the conventional ALPHA II FT-IR spectrometer from Bruker through the single reflection Eco-ATR module. All the data were collected with a resolution of $2 \mathrm{~cm}^{-1}$.

\section{FTIR and Vibrational Circular Dichroism (VCD) Spectroscopy}

The Infrared (IR), as well as the VCD spectra of the pellets, were recorded using an FTIR spectrometer (Vertex 70 Bruker) equipped with a VCD module (PMA 50 Bruker) consisting of a Photo-Elastic Modulator (PEM) and a fast acquisition dual channel. The IR spectrum was measured over the whole 800$3700 \mathrm{~cm}^{-1}$ range at a $2 \mathrm{~cm}^{-1}$ resolution while the VCD spectrum was obtained only in the $800-1800 \mathrm{~cm}^{-1}$ region. The alignment of the spectrometer was controlled by monitoring the mirror-image relation between the VCD spectra of the two enantiomers of camphor $\left(0.3 \mathrm{M}\right.$ in $\left.\mathrm{CCl}_{4}\right)$. The possible artefacts due to the $\mathrm{KBr}$ pellets birefringence were corrected by following the procedure proposes by Merten et al. derived from that introduced by Buffeteau et al. ${ }^{46} 47$ Briefly, it consists in rotating the sample in the plane perpendicular to the light propagation axis for each side (front $\mathrm{F}$ or back $\mathrm{B}$ ) at 0 and $90^{\circ}$. Therefore, each VCD spectrum is composed of the average between the four spectra recorded at the different crystal sample positions. Each spectrum shown hereafter corresponds to 4 hours acquisition time, i.e. one hour per orientation.

\section{Solid-State Synthesis}

Spontaneous cyclisation reaction was obtained by heating the $\mathrm{LL}$ and LD pellets to a temperature of $125^{\circ} \mathrm{C}$ for increasing times. ${ }^{27}$ The reproducibility of the experiment was checked by repeating the procedure on three different pellets, for $L L$ and LD, and verifying that the results are identical. The reaction was monitored by recording the IR spectra as a function of time. The spectra were taken before putting the pellets into the oven (time zero) then after $1 \mathrm{hr}, 4 \mathrm{hrs}, 9 \mathrm{hrs}$, and $21 \mathrm{hrs}$ in the oven. The reaction was considered to be completed when the IR spectrum did not evolve in time anymore. Then, a VCD spectrum was recorded as described above.

\section{Results and Discussion}

Infrared Spectroscopy of Solid-State c-LL and c-LD

\section{Mass Spectrum}


Figure 2 compares the IR absorption spectrum of $c-L L$ and c-LD diluted in $\mathrm{KBr}$ with the ATR spectrum of the pure powder, in the amide I and II regions. The similitude between the two spectra confirms that the $\mathrm{KBr}$ pellet and the pure solid are constituted of powder of identical nature, namely, microcrystals. The diastereomers especially differ in the amide I region: as reported before, C-LL shows a doublet at $1663-1677 \mathrm{~cm}^{-1}$, which contrasts to the single broadened band at $1677 \mathrm{~cm}^{-1}$ in c-LD. ${ }^{35}$ The doublet observed for the $\mathrm{v}(\mathrm{CO})$ stretch of $\mathrm{c}-\mathrm{LL}$ was assigned to bound and free $\mathrm{CO}$ groups within dimers involving a double $\mathrm{NH}$..OC bond, an assignment confirmed by VCD spectra. ${ }^{35}$ The single band observed in C-LD is characteristic of free CO groups. It is therefore likely that the crystal cohesion is mainly due to dispersion in C-LD. As expected for the symmetrical C-LD, no VCD signal was observed.

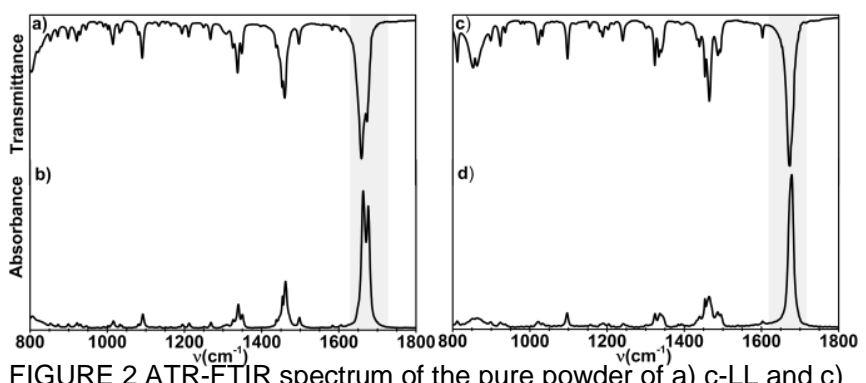

FIGURE 2 ATR-FTIR spectrum of the pure powder of a) c-LL and c) c-LD. FTIR absorption of the $\mathrm{KBr}$ pellet of b) c-LL and d) c-LD.

\section{Infrared Spectroscopy of Solid-State LL and LD}

Figure 3 compares the IR absorption of $L L$ and $L D$ in a $\mathrm{KBr}$ pellet with the ATR spectrum of the pure powder, in the fingerprint region. ${ }^{27}$ The ATR and IR absorption spectra are very similar, pointing at the polycrystalline nature of the samples. The ATR spectrum shown here strongly differs from that reported for the self-assembled nanotubes, which contain three well-defined transitions at 1256, 1389 and $1555 \mathrm{~cm}^{-1}$. These transitions are not observed here. Moreover, the bands $1143(A), 1205(B),(H)$ and $1732 \mathrm{~cm}^{-1}$ (I) present in the ATR spectrum reported here are absent in that of the nanostructures. ${ }^{19}$ These results allow us to rule out the existence of supramolecular nanostructures in the sample studied here. The IR absorption is congested and shows bands due to both neutral and zwitterion forms of the dipeptides. This is especially apparent for the $v(\mathrm{CO})$ stretch, which dominates the fingerprint region. First, two narrow features are observed at $1732 \mathrm{~cm}^{-1}$ and $1691 \mathrm{~cm}^{-1}$. The former is the $\mathrm{v}(\mathrm{CO})$ stretch of the carboxylic acid $\mathrm{COOH}$ and the latter the $\mathrm{v}(\mathrm{CO})$ stretch of the peptide bond. Second, an intense band is observed at $1676 \mathrm{~cm}^{-1}(\mathrm{G})$. It is assigned to the $\mathrm{v}(\mathrm{CO})$ stretch of the carboxylate anion $\mathrm{COO}^{-}$of the zwitterion. ${ }^{48}$ The ratio between the neutral vs. zwitterion contributions depends on the water content of the pellet and on the relative absolute configuration of the residues. Pellets left at ambient conditions are strongly hydrated, as attested by the broad bands at $~ 3440$ $\mathrm{cm}^{-1}$ and $\sim 1630 \mathrm{~cm}^{-1}$ due to $v_{3}$ and $v_{2}$ of water. In these conditions, the zwitterion dominates, for both LL and LD samples, which show almost identical spectra. For pellets kept 12 hours at $80^{\circ} \mathrm{C}$, the neutral and zwitterion contributions are of the same order of magnitude for LL. No effects of humidity are observed for LD that always shows a dominant contribution of the zwitterion.

Several transitions appear in the $\mathrm{NH}$ bend region at $1456 \mathrm{~cm}^{-1}$ (D), $1498 \mathrm{~cm}^{-1}(\mathrm{E})$, and $1566 \mathrm{~cm}^{-1}(\mathrm{~F})$. Apart from the latter, which is observed in dry LL only and is therefore assigned to the neutral dipeptides, the other bands are of limited intensity. They are not structure-dependent, nor are the intense aromatic and aliphatic $\mathrm{CH}$ bending modes at $1060-1280 \mathrm{~cm}^{-1}$. These bands are not very sensitive to the neutral or zwitterionic nature of the molecule, apart from that at $1271 \mathrm{~cm}^{-1}$ (C), which is characteristic of the neutral form. They are not sensitive either to the relative absolute configuration. Therefore, we will not consider them further.
The hydride stretch region, shown in Figure $\mathrm{S} 1$ of the Electronic Supplementary Information (ESI), is characterized by a broadband absorption between $2400 \mathrm{~cm}^{-1}$ and $2800 \mathrm{~cm}^{-1}$, attributed to a zwitterion, like observed in alanine diluted in $\mathrm{KBr}$ pellets. ${ }^{48}$

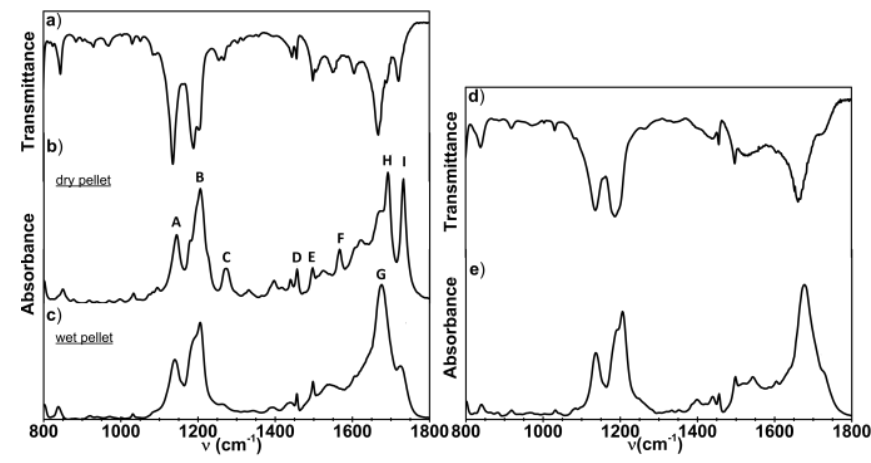

FIGURE 4 a) ATR-FTIR spectrum of the pure LL powder in the 800 $1800 \mathrm{~cm}^{-1}$ region. b) FTIR spectrum of LL diluted in a $\mathrm{KBr}$ pellet (dry pellet) c) same for a water-containing pellets. d) ATR-FTIR spectrum of the pure LD powder in the $800-1800 \mathrm{~cm}^{-1}$ region. e) FTIR spectrum of LD diluted in a $\mathrm{KBr}$ pellet.

Sharper bands at 3302 and $3173 \mathrm{~cm}^{-1}$ are assigned to the amide $\mathrm{v}(\mathrm{NH})$ stretches.

\section{Vibrational Circular Dichroism of Solid-State LL and LD}

Figure 4 reports the VCD spectra of $L L$ and $L D$ diluted in $\mathrm{KBr}$, as well as that of their enantiomers. A good mirror image relation is obtained between the spectra of enantiomers. Broad features in the IR absorption spectrum appear as multiplets in the VCD spectrum, which indicates that they correspond to superimposed

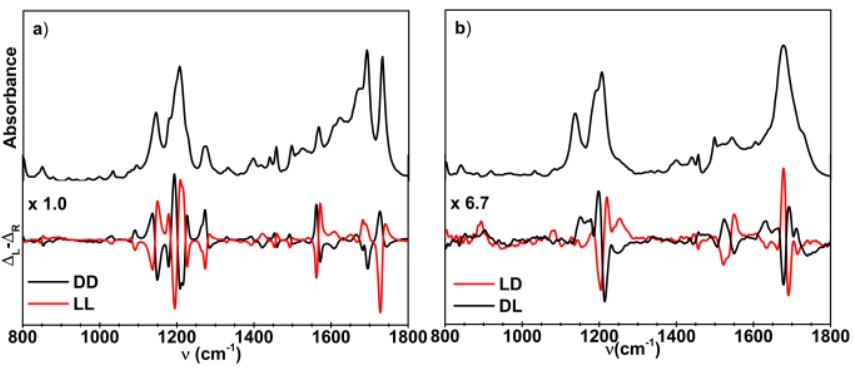

FIGURE 3 FTIR (top) and VCD (bottom) spectra in the fingerprint region of a) $L L$ and b) $L D$

IR transitions. The bands due to the neutral species at 1271 , 1566 and $1732 \mathrm{~cm}^{-1}$ are relatively intense in the VCD spectrum of LL. They all show a bisignate pattern. The $1060-1280 \mathrm{~cm}^{-1}$ region also shows strong VCD activity.

LD shows VCD intensities about 7 times weaker than LL. The $1060-1280 \mathrm{~cm}^{-1}$ is still the most active one, as it was in LL. The $1600-1800 \mathrm{~cm}^{-1}$ region is more complex than in LL.

The VCD spectra of $c-L L$ and $c-L D$ have been reported before. As expected for a symmetrical compound, the spectrum of $c-L D$ shows no activity.

\section{Formation of cyclo Diphenylalanine by a Thermal Reaction}

Both LD and LL IR absorption spectra evolve with heating time, which indicates their transformation into a product referred to as $h \mathrm{LL}$ and $h \mathrm{LD}$, where $h$ stands for 'heated'. The kinetics are identical for the three samples tested for a given diastereomer, but the thermal reaction is faster for LD than LL. The fact that the difference spectra (Figure S2 of the ESI) show bands that continuously increase while others steadily decrease with time suggests the absence of intermediate structures, at least on a measurable time scale, in the reactive process.

After completion of the thermal reaction, the mass spectrum recorded for electrosprayed solutions made from each pellet indicates the presence of cyclo diphenylalanine, as well as the complete disappearance of the parent linear dipeptide (see 
Figure $\mathrm{S} 3$ of the ESI). No peak corresponding to $\mathrm{CO}_{2}$ loss from the parent is observed, which indicates that DKP formation is the only product. This is in contrast to the same reaction involving phenylalanine on silica, for which $\mathrm{CO}_{2}$ loss was also observed. ${ }^{49}$ The mass spectra therefore suggest that $h \mathrm{LL}$ and $h \mathrm{LD}$ indeed are C-LL and c-LD.

The comparison between IR absorption spectra of the thermal reaction products, namely $h \mathrm{LL}$ and $h \mathrm{LD}$, and c-LL and c-LD confirms this hypothesis (Figure 5). The IR spectra are very similar indeed in terms of shape and position of the bands. However, the band assigned to the bound $v(\mathrm{CO})$ stretch is shifted down by $2 \mathrm{~cm}^{-1}$ in $h \mathrm{LL}$ relative to c-LL. C-LD and $h \mathrm{LD}$ also show small differences in that region. In C-LD, the band shows an asymmetry (frequency separation between the two free $v(C O)$ stretches) of $7 \mathrm{~cm}^{-1}$ while in $h L D$ it is $11 \mathrm{~cm}^{-1}$. These minor differences might reflect a difference in crystal packing.

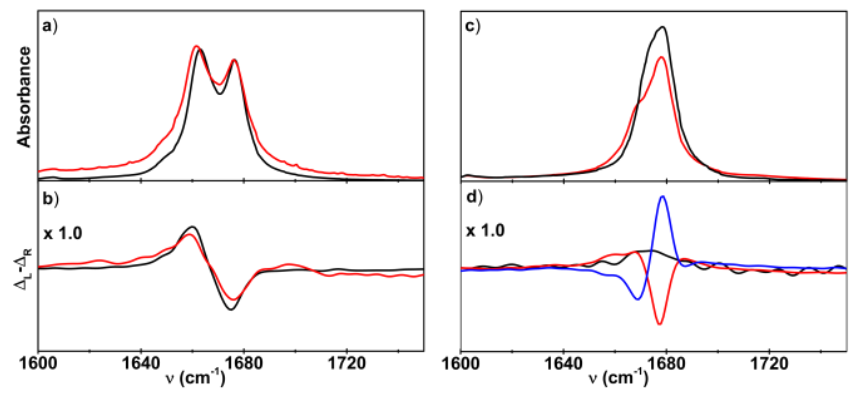

FIGURE 5 Comparison between the spectra of the reaction products $h \mathrm{LL}$ (red line) and c-LL (black line) in the region of the $\mathrm{v}(\mathrm{CO})$ stretch: a) IR absorption in absorbance (A) units and b) VCD spectra. Same comparison for the reaction products $h \mathrm{LD}$ (red line), $h \mathrm{DL}$ (blue line) and C-LD (black line): c) IR absorption and d) VCD spectra.

\section{Formation of a chiral phase}

\section{Achiral cyclo LD diphenylalanine.}

c-DL and C-LD purchased from the supplier exhibit identical IR absorption and do not possess any VCD activity. The lack of optical activity can be due to two reasons. First, c-LD could be a $\mathrm{C}_{\mathrm{i}}$ molecule, devoid of chirality due to the presence of a center of symmetry. ${ }^{39}$ In this case, c-LD and c-DL are identical non-chiral molecules. However, laser spectroscopy combined with quantum chemical calculations indicates that the $C_{i}$ structure is a transition state and that the most stable form in the gas phase is a non-symmetrical $C_{1}$ structure, with non-equivalent phenyl groups. One phenyl is indeed folded over the DKP ring while the other one is extended outwards. In what follows, we will denote the phenyl position by a subscript $F$ for folded and $E$ for extended. $c-L D(c-D L)$ is chiral because $c-L_{F} D_{E}\left(c-D_{F} L_{E}\right)$ is the mirror image of $c-L_{E} D_{F}\left(c-D_{E} L_{F}\right)$. As $c-L_{F} D_{E}$ and $c-D_{E} L_{F}$ are identical, we are left with two enantiomers $c-L_{F} D_{E}$ and $c-L_{E} D_{F}$. The interconversion between them proceeds through largeamplitude motions and involves low barriers. As a result, C-LD is a racemic mixture of two easily converting enantiomers, which explains its lack of VCD activity.

\section{Formation of chiral cyclo $L L$ and $D D$ diphenylalanine}

The VCD spectrum of $h \mathrm{LL}$ is the mirror image of that of $h \mathrm{DD}$. It is very similar to that of $\mathrm{C}-\mathrm{LL}$, notwithstanding the small broadening at the low-energy side, which was already apparent in the IR absorption. Similarly, the VCD spectrum of $h \mathrm{DD}$ compares well to that of C-DD. The VCD spectra, therefore, confirm the other experimental findings, namely the MS and FTIR results, that $h \mathrm{LL}$ is identical to c-LL and $h D D$ to $c-D D$. As peptide bond formation preserves the absolute configuration of the amino-acids chiral centers, VCD activity of the products is expected.

\section{Formation of chiral cyclo $L D$ and $D L$ diphenylalanine.}

VCD activity in $h$ LD is more surprising. The fact that $h$ LD shows optical activity, in contrast to c-LD, suggests that the crystal is chiral. Moreover, the VCD spectra of $h \mathrm{LD}$ and $h \mathrm{DL}$ show specular relation. Therefore, enantiomers are formed when starting from LD or DL. We can discuss these surprising results at the light of what is known on chiral supramolecular objects. These objects can first form from completely achiral sub-units assembled in a chiral manner. ${ }^{50}$ The formed system can be a conglomerate, in the absence of perturbation ${ }^{51}$ or can be driven into a given chirality by a chiral influence. This is the case for foldamers built from achiral monomers, the helicity of which is determined by the absolute configuration of chiral tartrate gemini. ${ }^{52}$ Second, it can form from non-permanently chiral subunits that synchronize their chirality. ${ }^{53,54}$ This is observed for 2-propyl-1-H-benzimidazole in the solid state. ${ }^{55}$ The molecular sub-unit is transiently chiral, although free rotation around a single bond allows fast interconversion between the enantiomers in solution. Still, the molecule crystallizes as a chiral crystal endowed with a chiroptical response. This is an example of chirality synchronization, a phenomenon that has been observed also under isolated conditions. ${ }^{5657}$ Surprisingly, always the same enantiomers of the 2-propyl-1-H-benzimidazole crystal is found whatever the experimental conditions. Our results contrast to the latter as enantiomeric crystals are formed when reacting enantiomeric reagents. This can be explained by considering the possible cyclization mechanism in the $\mathrm{KBr}$ pellet, leading to the DKP formation from the linear dipeptide. The proposed mechanism in solution involves neutral dipeptide. ${ }^{43}$ The first step is the isomerization of the amide, from trans to cis geometry of the peptide bond ${ }^{58}$ as confirmed by theoretical studies. ${ }^{59,60}$ This allows nucleophilic addition of the amino group to the carbonyl carbon atom and then the loss of water to form the DKP ring. It was also noticed that the dipeptide with alternate absolute configuration shows larger cyclization propensity due to steric effects, the LD configuration of the DKP involves indeed substituents on opposite sides of the DKP ring. ${ }^{58}$ This is in line with the experimental findings described here that LD cyclizes more rapidly than LL.

\section{Symmetry consideration.}

A possible interpretation of the observed effect rests upon the symmetry of the reagent. Although both residues are indiscernible in the product, they are non-symmetrical in the reagent, because one of them is on the $C$ terminus and the other one on the $\mathrm{N}$ terminus. The most stable calculated structures of jet-cooled LL and LD, to which the experimentally-observed species were assigned, involve a bifurcated hydrogen bond with the amide $\mathrm{NH}$ interacting with both the $\mathrm{NH}_{2}$ terminus and the acid $\mathrm{CO}$, see Figure 6 . The phenyl rings are in a T-shape arrangement. $^{22}$ The isomerization of the amide may involve rotation around the amide bond. If the reaction proceeds in solution or on resin, the amide rotation is accompanied by free rotation of the benzyl substituents. As a result, the chiral centers lose the memory of their origin, in other words, whether they were located on the $\mathrm{N}$ or $\mathrm{C}$ terminus. A racemic mixture of $\mathrm{C}$ $L_{F} D_{E}$ and $C-L_{E} D_{F}$ is obtained resulting in zero VCD signal. If the reaction is conducted in the $\mathrm{KBr}$ pellets, the phenyl rotation is not free in the solid and each phenyl keeps a selected orientation depending on the terminus it belongs to. We will use as an example the most stable form of DL where $D$ is the $N$ terminus (see Figure 6 ). In this structure, the phenyl on the $D$ residue is folded over the peptide backbone and the $L$ is directed outside. Rotating the amide bond and conducting the reaction without rotating the benzyl substituents results in $C-D_{F} L_{E}$. Conversely, starting from the same geometry for $L D$ results to $C-L_{F} D_{E}$, which is enantiomer to $c-D_{F} L_{E}$. This reaction illustrates well the Curie principle postulated in 1894 saying that "the symmetries of the causes are to be found in the effects".

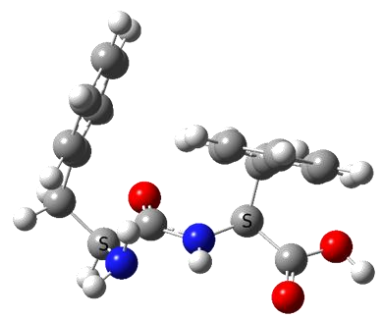

LL

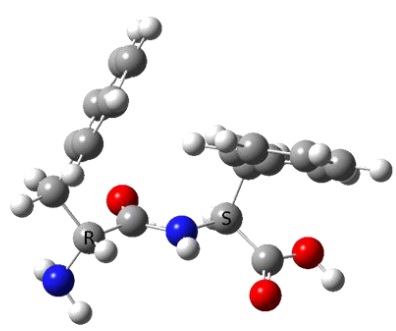

DL 
FIGURE 6 Most stable geometry of LL and DL in the gas phase

The comparison between the experimental VCD spectra, in the amide I region, and those simulated in the gas-phase for the c$L_{F} D_{E}$ and $C-D_{F} L_{E}$ enantiomers is shown in Figure 7. The whole spectrum is shown in Figure S4 of the Supplementary Information. The calculated spectrum corresponds to the previously published $B$ conformer, which is the second most stable conformer in the gas phase. ${ }^{22}$ Briefly, the spectrum was calculated in the frame of the density functional theory at the $B 3 L Y P / 6-311++g(d, p)$ level, including the D3 empirical dispersion term, using the Gaussian 09 software. ${ }^{61}$ The calculated frequencies were empirically scaled by 0.952 to make the experimental and calculated $v(\mathrm{CO})$ match. This unusually large scaling factor accounts not only for anharmonicity and basis set incompleteness, but also for the fact that we use the spectrum of a monmer to reproduce that of a crystal. Despite this approach being justified by the fact that the crystal is a dispersion driven structure, deprived of $\mathrm{NH}$... O hydrogen bonds, this is still a crude approximation, which explains the deficiencies of the calculated spectrum, in particular the overestimated gap between $v(\mathrm{CO})$ and $\beta(\mathrm{NH})$ bands. However, a good agreement is obtained between experiment and calculation in the $v(C O)$ region and none of the other calculated conformers of $c$-LD accounts for the experimental spectrum. ${ }^{35}$ This argument reinforces our conclusion concerning the formation of a chiral phase resulting from chirality synchronization between transiently chiral sub-units similar to that shown in Figure 7.
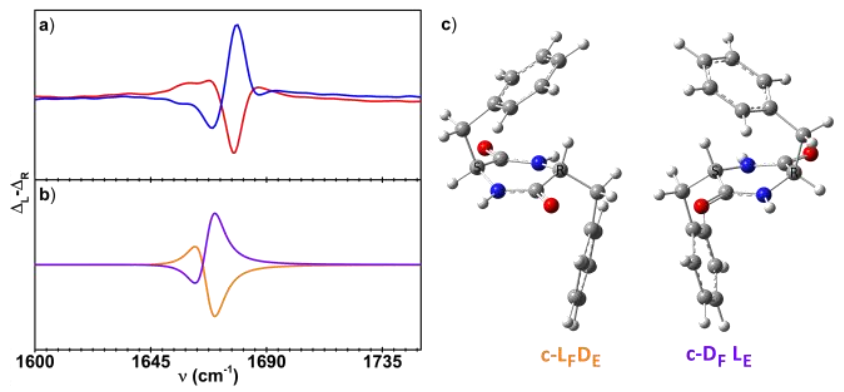

FIGURE 7 a) Experimental VCD spectrum of $h$ LD (red line) and $h D L$ (blue line) . b) Simulated VCD spectrum of $c-L_{F} D_{E}$ (orange line) and $C-D_{F} L_{E}$ (violet line). c) Corresponding calculated structures.

\section{Conclusion}

The comparison between ATR and IR absorption spectra shows that the pure solid and the sample diluted in $\mathrm{KBr}$ pellets have identical structures. C-LL and C-LD differ in their packing. While the c-LL powder is composed of hydrogen-bonded dimers, the cohesion of c-LD crystals is mainly due to dispersion. IR spectroscopy clearly indicates that the samples of LL and LD linear dipeptides studied here are not composed of nanotubes. $\mathrm{LL}$ is composed of a mixture of zwitterion and neutral forms of the peptide; whose relative contributions depend on the water content of the sample. In contrast, LD mainly exists as a zwitterion. In situ synthesis of cyclo diphenylalanine is easily achieved by heating the parent linear dipeptides at a temperature of $\sim 125^{\circ} \mathrm{C}$. Interestingly, the C-LD DKP dipeptide formed thereby shows optical activity. Moreover, the cyclic dipeptides formed from the LD and DL parents are enantiomers and show opposite VCD spectra. This is tentatively explained in terms of chirality synchronization between the monomers within the crystal, which retain the chirality of the reagent. Besides providing information on the structure of the solid, VCD experiments allowed, therefore, evidencing a new chiral phase made from a transiently chiral molecule.

\section{Acknowledgements}

We thank Mr. Antoine Béthune and Mr. Jérémy Lefèbvre for experimental assistance.

\section{Supporting information}

Additional supporting information may be found in the online version of this article at the publisher's website. 


\section{REFERENCES AND NOTES}

1. Guo C, Luo Y, Zhou R, Wei G. Triphenylalanine peptides self-assemble into nanospheres and nanorods that are different from the nanovesicles and nanotubes formed by diphenylalanine peptides. Nanoscale 2014;6(5):28002811.

2. Joshi KB, Verma S. Participation of aromatic side chains in diketopiperazine ensembles. Tetrahedron Letters 2008;49(27):4231-4234.

3. Manchineella S, Govindaraju T. Molecular Self-Assembly of Cyclic Dipeptide Derivatives and Their Applications. Chempluschem 2017;82(1):88-106.

4. Jeon J, Shell MS. Self-Assembly of Cyclo-diphenylalanine Peptides in Vacuum. Journal of Physical Chemistry B 2014;118(24):6644-6652.

5. Jeziorna A, Stopczyk K, Skorupska E, Luberda-Durnas K Oszajca M, Lasocha W, Gorecki M, Frelek J, Potrzebowski MJ. Cyclic Dipeptides as Building Units of Nano- and Microdevices: Synthesis, Properties, and Structural Studies. Crystal Growth \& Design 2015;15(10):5138-5148.

6. Amaral HR, Kogikoski S, Silva ER, Souza JA, Alves WA. Micro- and nano-sized peptidic assemblies prepared via solid-vapor approach: Morphological and spectroscopic aspects. Materials Chemistry and Physics 2012;137(2):628-636.

7. Amdursky N, Molotskii M, Gazit E, Rosenman G. Elementary Building Blocks of Self-Assembled Peptide Nanotubes. Journal of the American Chemical Society 2010;132(44):15632-15636

8. Mossou E, Teixeira SCM, Mitchell EP, Mason SA, AdlerAbramovich L, Gazit E, Forsyth VT. The self-assembling zwitterionic form of L-phenylalanine at neutral $\mathrm{pH}$. Acta Crystallographica Section C 2014;70(3):326-331.

9. Lee JS, Yoon I, Kim J, Ihee H, Kim B, Park CB. SelfAssembly of Semiconducting Photoluminescent Peptide Nanowires in the Vapor Phase. Angewandte ChemieInternational Edition 2011;50(5):1164-1167.

10. Kogikoski S, Sousa CP, Liberato MS, Andrade-Filho T, Prieto T, Ferreira FF, Rocha AR, Guha S, Alves WA. Multifunctional biosensors based on peptidepolyelectrolyte conjugates. Physical Chemistry Chemical Physics 2016;18(4):3223-3233.

11. Silva RF, Araújo DR, Silva ER, Ando RA, Alves WA. IDiphenylalanine Microtubes As a Potential Drug-Delivery System: Characterization, Release Kinetics, and Cytotoxicity. Langmuir 2013;29(32):10205-10212.

Adler-Abramovich L, Reches M, Sedman VL, Allen S, Tendler SJB, Gazit E. Thermal and Chemical Stability of Diphenylalanine Peptide Nanotubes: Implications for Nanotechnological Applications. Langmuir 2006;22(3):1313-1320

13. Guo C, Luo Y, Zhou R, Wei G. Probing the Self-Assembly Mechanism of Diphenylalanine-Based Peptide Nanovesicles and Nanotubes. ACS Nano 2012;6(5):39073918.
14. Jeon J, Mills CE, Shell MS. Molecular Insights into Diphenylalanine Nanotube Assembly: All-Atom Simulations of Oligomerization. The Journal of Physical Chemistry B 2013;117(15):3935-3943

15. Andrade-Filho T, Martins TC, Ferreira FF, Alves WA Rocha AR. Water-driven stabilization of diphenylalanine nanotube structures. Theoretical Chemistry Accounts 2016;135(8):185.

16. Andrade-Filho T, Ferreira FF, Alves WA, Rocha AR. The effects of water molecules on the electronic and structural properties of peptide nanotubes. Physical Chemistry Chemical Physics 2013;15(20):7555-7559.

17. Rissanou AN, Georgilis E, Kasotakis E, Mitraki A Harmandaris V. Effect of Solvent on the Self-Assembly of Dialanine and Diphenylalanine Peptides. The Journal of Physical Chemistry B 2013;117(15):3962-3975

18. Azuri I, Adler-Abramovich L, Gazit E, Hod O, Kronik L Why Are Diphenylalanine-Based Peptide Nanostructures so Rigid? Insights from First Principles Calculations. Journal of the American Chemical Society 2014;136(3):963-969.

19. Görbitz $\mathrm{CH}$. Nanotube Formation by Hydrophobic Dipeptides. Chemistry - A European Journal 2001;7(23):5153-5159.

20. Goerbitz $\mathrm{CH}$. The structure of nanotubes formed by diphenylalanine, the core recognition motif of Alzheimer's beta-amyloid polypeptide. Chemical Communications 2006(22):2332-2334.

21. Im S, Jang SW, Lee S, Lee Y, Kim B. Arginine zwitterion is more stable than the canonical form when solvated by a water molecule. Journal of Physical Chemistry A 2008;112(40):9767-9770

22. Perez-Mellor A, Alata I, Lepere V, Zehnacker A. Chirality effects in the structures of jet-cooled bichromophoric dipeptides. Journal of Molecular Spectroscopy 2018:349:71-84

23. Gloaguen E, Valdes H, Pagliarulo F, Pollet R, Tardivel B, Hobza P, Piuzzi F, Mons M. Experimental and Theoretical Investigation of the Aromatic-Aromatic Interaction in Isolated Capped Dipeptides. The Journal of Physical Chemistry A 2009;114(9):2973-2982.

24. Borthwick AD. 2,5-Diketopiperazines: Synthesis Reactions, Medicinal Chemistry, and Bioactive Natural Products. Chemical Reviews 2012;112(7):3641-3716.

25. Basiuk VA, Gromovoy TY. THE GAS SOLID-PHASE 2,5 DIOXOPIPERAZINE SYNTHESIS - CYCLIZATION OF VAPOROUS DIPEPTIDES ON SILICA SURFACE. Collection of Czechoslovak Chemical Communications 1994;59(2):461-466

26. Ziganshin MA, Safiullina AS, Gerasimov AV, Ziganshina SA, Klimovitskii AE, Khayarov KR, Gorbatchuk VV Thermally Induced Self-Assembly and Cyclization of ILeucyl-I-Leucine in Solid State. The Journal of Physical Chemistry B 2017;121(36):8603-8610.

27. Ziganshin MA, Gerasimov AV, Ziganshina SA, Gubina NS Abdullina GR, Klimovitskii AE, Gorbatchuk VV, Bukharaev AA. Thermally induced diphenylalanine cyclization in solid 
phase. Journal of Thermal Analysis and Calorimetry 2016;125(2):905-912.

Jaworska M, Jeziorna A, Drabik E, Potrzebowski MJ. Solid State NMR Study of Thermal Processes in Nanoassemblies Formed by Dipeptides. Journal of Physical Chemistry C 2012;116(22):12330-12338.

Sinha S, Srivastava R, De Clereq E, Singh RK. Synthesis and antiviral properties of arabino and ribonucleosides of 1,3-dideazaadenine, 4-nitro-1,3-dideazapurine and diketopiperazine. Nucleosides Nucleotides \& Nucleic Acids 2004;23(12):1815-1824.

30. Grande F, Garofalo A, Neamati N. Small molecules antiHIV therapeutics targeting CXCR4. Current Pharmaceutical Design 2008;14(4):385-404.

31. Walchshofer N, Sarciron ME, Garnier F, Delatour $P$, Petavy AF, Paris J. Anthelmintic activity of 3,6-dibenzyl2,5-dioxopiperazine, cyclo(L-Phe-L-Phe). Amino Acids 1997;12(1):41-47.

Danda $\mathrm{H}$. Essential factors in asymmetric hydrocyanation catalyzed by cyclo (-(R)-Phe-(R)-His-). Synlett 1991;1991(04):263-264

Tanaka K, Mori A, Inoue S. The cyclic dipeptide cyclo [(S)phenylalanyl-(S)-histidyl] as a catalyst for asymmetric addition of hydrogen cyanide to aldehydes. The Journal of organic chemistry 1990;55(1):181-185.

34. Gdaniec M, Liberek B. STRUCTURE OF CYCLO(-LPHENYLALANYL-L-PHENYLALANYL-). Acta Crystallographica Section C-Crystal Structure Communications 1986;42:1343-1345.

Perez-Mellor A, Zehnacker A. Vibrational circular dichroism of a 2,5-diketopiperazine (DKP) peptide: Evidence for dimer formation in cyclo LL or LD diphenylalanine in the solid state. Chirality 2017;29(2):8996.

36. Garcia AM, Iglesias D, Parisi E, Styan KE, Waddington LJ, Deganutti C, De Zorzi R, Grassi M, Melchionna M, Vargiu AV and others. Chirality Effects on Peptide Self-Assembly Unraveled from Molecules to Materials. Chem 2018;4(8):1862-1876.

37. Ozawa $\mathrm{Y}$, Sato H, Kayano $\mathrm{Y}$, Yamaki N, Izato $\mathrm{Y}$, Miyake A, Naito A, Kawamura I. Self-assembly of tripeptides into c-turn nanostructures. Physical Chemistry Chemical Physics 2019;21(21):10879-10883.

38. Abo-Riziq AG, Bushnell JE, Crews B, Callahan MP, Grace $\mathrm{L}$, de Vries MS. Discrimination between diastereoisomeric dipeptides by IR-UV double resonance spectroscopy and ab initio calculations. International Journal of Quantum Chemistry 2005;105(4):437-445

39. Pérez Mellor A, Zehnacker A. Chirality Effects in JetCooled Cyclic Dipeptides. In: Ebata T, Fujii M, editors. Physical Chemistry of Cold Gas-Phase Functional Molecules and Clusters. Singapore: Springer; 2019. p 6387.

40. Alata I, Perez-Mellor A, Ben Nasr F, Scuderi D, Steinmetz $V$, Gobert F, Jaidane NE, Zehnacker-Rentien A. Does the Residues Chirality Modify the Conformation of a CycloDipeptide? Vibrational Spectroscopy of Protonated Cyclo- diphenylalanine in the Gas Phase. Journal of Physical Chemistry A 2017;121(38):7130-7138.

41. Dunbar RC, Steill JD, Oomens J. Chirality-Induced Conformational Preferences in Peptide-Metal Ion Binding Revealed by IR Spectroscopy. Journal of the American Chemical Society 2011;133(5):1212-1215.

42. Lepere V, Le Barbu-Debus K, Clavaguera C, Scuderi D, Piani G, Simon A-L, Chirot F, MacAleese L, Dugourd P, Zehnacker A. Chirality-dependent structuration of protonated or sodiated polyphenylalanines: IRMPD and ion mobility studies. Physical Chemistry Chemical Physics 2016;18(3):1807-17.

43. Moss $\mathrm{J}$, Bundgaard $\mathrm{H}$. Kinetics And Mechanism Of The Facile Cyclization of Histidyl-Prolineamide To Cyclo (HisPro) In Aqueous-Solution and The Competitive Influence of Human Plasma. Journal of Pharmacy and Pharmacology 1990;42(1):7-12.

44. Capasso S, Vergara A, Mazzarella L. Mechanism of 2,5dioxopiperazine formation. Journal of the American Chemical Society 1998;120(9):1990-1995.

45. Szardenings AK, Burkoth TS, Lu HH, Tien DW, Campbell DA. A simple procedure for the solid phase synthesis of diketopiperazine and diketomorpholine derivatives. Tetrahedron 1997;53(19):6573-6593.

46. Merten C, Kowalik T, Hartwig A. Vibrational circular dichroism spectroscopy of solid polymer films: Effects of sample orientation. Applied Spectroscopy 2008;62(8):901905.

47. Buffeteau T, Lagugne-Labarthet FS, Sourisseau C Vibrational circular dichroism in general anisotropic thin solid films: Measurement and theoretical approach. Applied Spectroscopy 2005;59(6):732-745

48. Cao XL, Fischer G. Infrared spectra of monomeric Lalanine and $\mathrm{L}$-alanine- $\mathrm{N}-\mathrm{d}(3)$ zwitterions isolated in a $\mathrm{KBr}$ matrix. Chemical Physics 2000;255(2-3):195-204.

49. Basiuk VA, Gromovoy TY, Glukhoy AM, Golovaty VG Chemical-Transformations of Proteinogenic Amino-Acids During their Sublimation In the Presence of Silica. Origins of Life and Evolution of the Biosphere 1991;21(3):129-144.

50. Kama AB, Genois R, Massuyeau F, Sidibe M, Diop CAK Gautier R. Cyclohexylammonium sulfanilate: A simple representative of the chiral materials containing only achiral building units. Materials Letters 2019;241:6-9.

51. Sunatsuki $\mathrm{Y}$, Fujita $\mathrm{K}$, Maruyama $\mathrm{H}$, Suzuki $\mathrm{T}$, Ishida $\mathrm{H}$, Kojima M, Glaser R. Chiral Crystal Structure of a P2(1)2(1)2(1) Kryptoracemate Iron(II) Complex with an Unsymmetric Azine Ligand and the Observation of Chira Single Crystal Circular Dichroism. Crystal Growth \& Design 2014;14(8):3692-3695.

52. Brizard A, Aime C, Labrot T, Huc I, Berthier D, Artzner F, Desbat B, Oda R. Counterion, temperature, and time modulation of nanometric chiral ribbons from geminitartrate amphiphiles. Journal of the American Chemical Society $2007 ; 129(12): 3754-3762$

53. Chang BS, Li X, Sun TL. Self-assembled chiral materials from achiral components or racemates. European Polymer Journal 2019;118:365-381. 
54

Gautier R, Klingsporn JM, Van Duyne RP, Poeppelmeier KR. Optical activity from racemates. Nature Materials 2016;15(6):591-592.

55. Quesada-Moreno MM, Cruz-Cabeza AJ, Aviles-Moreno JR, Cabildo P, Claramunt RM, Alkorta I, Elguero J, Zuniga FJ, Lopez-Gonzalez JJ. The Curious Case of 2-Propy1$1 \mathrm{H}$-benzimidazole in the Solid State: An Experimental and Theoretical Study. Journal of Physical Chemistry A 2017;121(30):5665-5674.

56. Asselin P, Madebene B, Soulard P, Georges R, Goubet M, Huet TR, Pirali O, Zehnacker-Rentien A. Competition between inter- and intra-molecular hydrogen bonding: An infrared spectroscopic study of jet-cooled amino-ethanol and its dimer. Journal of Chemical Physics 2016;145(22).

57. Oswald S, Seifert NA, Bohle F, Gawrilow M, Grimme S, Jager W, Xu YJ, Suhm MA. The Chiral Trimer and a Metastable Chiral Dimer of Achiral Hexafluoroisopropanol: A Multi-Messenger Study. Angewandte ChemieInternational Edition 2019;58(15):5080-5084.

58.

Bodansky M. Principles of Peptides Synthesis. Berlin: Springer Verlag; 1984.

59.

Zhu YY, Tang MS, Shi XY, Zhao YF. Quantum chemical study of cyclic dipeptides. International Journal of Quantum Chemistry 2007;107(3):745-753.
60.

Xia P, Wang C, Qi C. Theoretical Study on the Cyclization Mechanism of Dipeptides. Chinese Journal of Chemistry 2013;31(6):813-818

61. M. J. Frisch GWT, H. B. Schlegel, G. E. Scuseria, M. A Robb, J. R. Cheeseman, G. Scalmani, V. Barone, B. Mennucci, G. A. Petersson, H. Nakatsuji, M. Caricato, X. Li, H. P. Hratchian, A. F. Izmaylov, J. Bloino, G. Zheng, J. L. Sonnenberg, M. Hada, M. Ehara, K. Toyota, R. Fukuda, J. Hasegawa, M. Ishida, T. Nakajima, Y. Honda, O. Kitao, H. Nakai, T. Vreven, J. A. Montgomery, J. E. Peralta, F. Ogliaro, M. Bearpark, J. J. Heyd, E. Brothers, K. N. Kudin, V. N. Staroverov, R. Kobayashi, J. Normand, K Raghavachari, A. Rendell, J. C. Burant, S. S. Iyengar, J. Tomasi, M. Cossi, N. Rega, J. M. Millam, M. Klene, J. E. Knox, J. B. Cross, V. Bakken, C. Adamo, J. Jaramillo, R. Gomperts, R. E. Stratmann, O. Yazyev, A. J. Austin, R. Cammi, C. Pomelli, J. W. Ochterski, R. L. Martin, K. Morokuma, V. G. Zakrzewski, G. A. Voth, P. Salvador, J. J. Dannenberg, S. Dapprich, A. D. Daniels, Farkas, J. B. Foresman, J. V. Ortiz, J. Cioslowski, D. J. Fox. Gaussian 09, Revision B.01. 2010.

Graphical Abstract
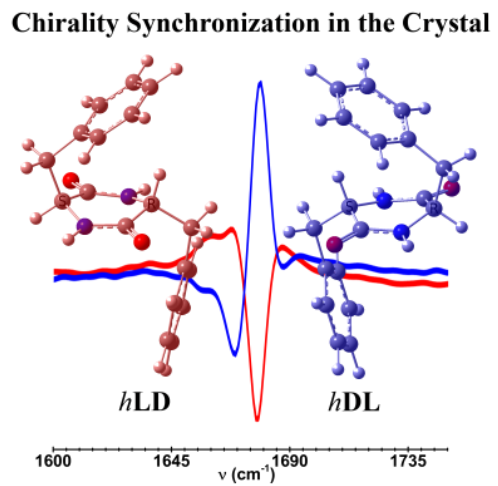